\title{
Alterations in optical coherence tomography angiography findings in patients with high myopia
}

\author{
Turgay Ucak $\mathbb{D}^{1} \cdot$ Erel Icel $\mathbb{D}^{1} \cdot$ Hayati Yilmaz $\mathbb{D}^{1} \cdot$ Yucel Karakurt $^{1} \cdot$ Gamze Tasli $^{1} \cdot$ Adem Ugurlu $\mathbb{D}^{1} \cdot$ Erdinc Bozkurt ${ }^{2}$
}

Received: 19 November 2019 / Revised: 8 February 2020 / Accepted: 12 February 2020 / Published online: 24 February 2020

(c) The Author(s), under exclusive licence to The Royal College of Ophthalmologists 2020

\begin{abstract}
Purpose The aim of this study was to determine the macular changes using optical coherence tomography (OCT) and OCTangiography (OCT-A) in eyes with high myopia. Determining the alterations in vascular structures can provide a clearer understanding of the pathophysiological mechanisms of this disease and help define new treatment options and preventive measures.

Materials and methods Ninety-two patients with high myopia (axial length $\geq 26 \mathrm{~mm}$ ) and 70 control cases without any known systemic or ocular diseases were enrolled in this prospective study. One eye of each patient was included in the statistical analyses.

Results Retinal nerve fiber layer (RNFL) thickness and Early Treatment Diabetic Retinopathy Study (ETDRS) macula map values were lower in myopia compared with the controls. Both superior and inferior ganglion cell complex (GCC) thicknesses were significantly thinner in the high myopia compared with the controls $(p<0.001)$. Regarding the OCT-A findings, although superficial or deep foveal avascular zones (FAZ) did not significantly differ between the two groups, the density values of superficial and deep microvessels were significantly lower in the high myopia group compared with the control cases.

Conclusions In patients with high myopia, with an increase in the axial length and a decrease in RNFL and GCC thicknesses, the vascular densities of the superficial and deep retina were reduced in the macular region.
\end{abstract}

\section{Introduction}

High myopia is a significant cause of permanent visual impairment, affecting particularly younger persons in all over the world. It has been defined as a refractive error with a spherical equivalent exceeding -6 diopters and/or an axial length (AL) longer than $26 \mathrm{~mm}$. The main pathophysiological mechanism of high myopia is an excessive axial elongation, which causes chorioretinal stretching and thinning. The subsequent thinning of the chorioretinal tissue is associated with poor blood circulation that may result in neovascularization [1-4].

Turgay Ucak

turgayucak10@gmail.com

1 Department of Ophthalmology, Faculty of Medicine, Erzincan University, Erzincan, Turkey

2 Department of Ophthalmology, Faculty of Medicine, Kafkas University, Kars, Turkey
Many ocular changes have been reported in high myopic eyes, including patchy or diffuse chorioretinal atrophy, thinning in the retinal nerve fiber layer (RNFL), macular atrophy, macular hole formation, and a dome-shaped macula, together with alterations in the retinal vessel morphology [5-7].

High myopia can affect the macula, leading to a dramatic reduction in visual acuity. Therefore, it is highly important for ophthalmologists to determine macular changes associated with high myopia. Optical coherence tomography (OCT) and OCT-angiography (OCT-A) are astonishing tools for the identification of macular pathologies and follow-up of patients. OCT-A is an advanced ophthalmic imaging technique that can evaluate the vessel network in different layers of retinal structures without any requirement for contrast agents [8].

Studies investigating the use of different OCT-A instruments suggest that it is almost impossible to make a comparison between them [9]. The features of OCT-A findings have already been described for the high myopia using other commercially available OCT-A devices [10], however, 
there is no study with Nidek's RS3000 Advance OCT-A device about high myopia. Since high myopia can dramatically damage visual acuity, the findings of OCT-A are important to elucidate the pathophysiological mechanisms and each device should have its own database. The aim of the present study was to analyse the macular changes using OCT and OCT-A in eyes with high myopia using the Nidek's RS300 Advance device. In that aspect, identification of the alterations in vascular structures can assist us to more clearly understand the pathophysiological mechanisms of the disease and describe novel treatment modalities and protective measures.

\section{Patients and method}

Ninety-two eyes of 92 adult patients with high myopia $(\mathrm{AL} \geq 26 \mathrm{~mm}$ ) who were being followed up in our hospital and 70 eyes of 70 control cases without any known systemic or ocular diseases were enrolled in this prospective study. A broad ocular examination, including the measurement of visual acuity (LogMAR converted), analysing intraocular pressure (IOP) using Goldmann applanation tonometry, and fundus examination with a 90-diopter noncontact lens, was performed on each eye. The AL and biometric measurements were undertaken using a Nidek AL-Scan device (Nidek CO., Gamagori, Japan). Exclusion criteria were as follows; patients with a history of any ocular diseases, such as retinal vascular occlusion, glaucoma, or uveitis, those with a history of previous trauma, and the patients with chronic systemic diseases such as diabetes mellitus or hypertension. We excluded patients who underwent ocular surgery, including LASIK and ICL, in both the control and high myopia groups. The individuals with a best-corrected visual acuity (BCVA) > 0.3 (LogMAR) and patients with astigmatism higher than \pm 3 diopters were also excluded. If choroid scleral junction could not be clearly defined on OCT, the patients were also not included in the study. The International Photographic Classification and Grading System for Myopic Maculopathy was applied in identification of fundus changes. Regarding this grading system, only patients with category 0 (no myopic retinal degenerative lesion) and category 1 (tessellated fundus) were included in the study $[11,12]$. We excluded diffuse chorioretinal atrophy, patchy chorioretinal atrophy, macular atrophy, and posterior staphyloma because of poor OCT-A image quality. Informed consent was obtained from all participants. The study was approved by the Ethics Committee of Erzincan University.

OCT images were obtained using the Nidek RS3000 Advance system. The images were retrieved from four radial scan lines, cantered at the fixation point, at the angles of $0^{\circ}, 45^{\circ}, 90^{\circ}$, and $135^{\circ}$. The RNFL thickness (superior, nasal, inferior, and temporal) and the superior and inferior ganglion cell complex (GCC) thicknesses were recorded. Retinal thicknesses measurements (nine regions) defined by the Early Treatment Diabetic Retinopathy Study (ETDRS) were also recorded [13].

The retinal microvascular network was identified using OCT-A (Nidek RS3000 Advance). The scanning was repeated if the signal strength index (SSI) quality was $<7 /$ 10 . The center of the foveal avascular zone (FAZ) was determined by analysing the intensity gradient of the image from center to the periphery. The scanning area used in this study was $3 \times 3 \mathrm{~mm}$, centered on the fovea. Superficial and deep vascular plexus angiograms were obtained. The superficial vascular plexus was extending from the internal limiting membrane to the inner plexiform layer, while the deep vascular plexus was lying from the inner nuclear layer to the outer plexiform layer (Fig. 1) [14]. All the numerical values in the study obtained automatically. Segmentation algorithm automatically was chosen by the device. Some patients were excluded because of poor quality scans due to various factors such as reduced signal strength due to ocular media opacity, and image quality limitations of the device. We also removed patients with motion artefacts, segmentation errors, and projection artefacts in the images. All the data assessed from the OCT-A images were reanalysed due to correct the magnification in the myopic eyes with the Bennett's formula. The Bennett's method using the AL as the main correction factor is more accurate than the method using keratometry [15].

\section{Statistical analyses}

Statistical analysis was performed using the SPSS statistical software package for Windows (version 21.0, SPSS, Inc., Chicago, IL). Power calculation was not calculated as the study was exploratory. The numerical data were presented with mean \pm standard deviation. The Shapiro-Wilk test was used to determine whether the sample came from a normally distributed population. According to the normality test results, the comparison of the numerical data between the two groups was performed using the independent samples $t$ test or Mann-Whitney $U$ test. The gender differences in the two groups were compared using the Pearson chi-square test. The relationship between the superficial (SVD) and deep retinal vascular densities (DVD) and age, AL, RNFL thickness, BCVA, and GCC thickness were assessed using Pearson's correlation analysis. After checking the collinearity statistics, the multivariate linear regression analysis was performed to determine the independent associations for 


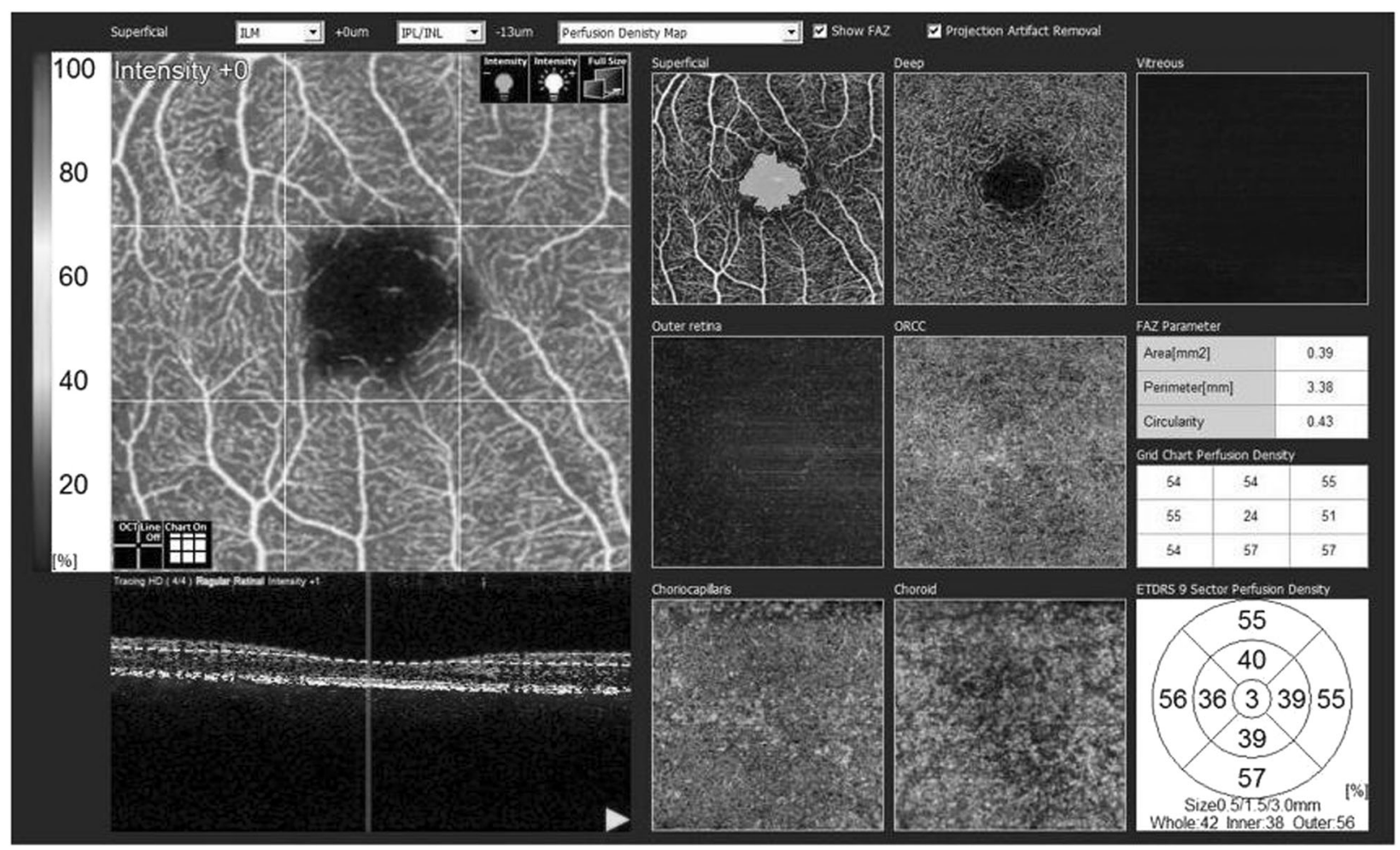

Fig. 1 We used Nidek's RS-3000 Advance and Navis Ex. Ver. 1.1.5 software to collect and analyze SD-OCT and OCT-A images. The FAZ metrics were autodetected by the AngioScan software. The software only enables to calculate the FAZ metrics at the level of superficial capillary plexus.

Table 1 Demographic features of study participants.

\begin{tabular}{llll}
\hline & $\begin{array}{l}\text { High myopia } \\
(n: 92)\end{array}$ & $\begin{array}{l}\text { Control } \\
(n: 70)\end{array}$ & $p$ \\
\hline Age (years) & $35.19 \pm 14.29$ & $36.22 \pm 11.42$ & 0.55 \\
Male/female & $36 / 56$ & $28 / 42$ & 0.11 \\
\hline
\end{tabular}

the SVD and DVD. A $p$ value of $<0.05$ was considered statistically significant.

\section{Results}

The demographic features of the participants are summarized in Table 1. There was no significant difference between the two groups regarding age $(p=0.55)$ or gender $(p=0.11)$. The ocular findings of the participants are presented in Table 2. The mean IOP values were similar between the high myopia and control groups. The logMAR converted BCVA was significantly greater in high myopia patients $(p<0.001)$. However, as expected, the spherical equivalent $(p<0.001)$ and $\mathrm{AL}(p<0.001)$ significantly differed between the two groups. Central macular thicknesses (CMT) were similar between the groups $(p=0.29)$. High myopia group was having significantly thinner superior and inferior GCC values
Table 2 Ocular findings of study participants.

\begin{tabular}{lccc}
\hline & $\begin{array}{l}\text { High myopia } \\
(n: 92)\end{array}$ & \multicolumn{1}{l}{$\begin{array}{l}\text { Control } \\
(n: 70)\end{array}$} & $p$ \\
\hline BCVA (LogMAR) & $0.049 \pm 0.079$ & $0.00 \pm 0.00$ & $<\mathbf{0 . 0 0 1}$ \\
IOP (mmHg) & $11.74 \pm 1.58$ & $11.74 \pm 1.03$ & 0.81 \\
Spherical equivalent & $-8.13 \pm 1.71$ & $0.53 \pm 0.27$ & $<\mathbf{0 . 0 0 1}$ \\
(Diopter) & & & \\
AL $(\mathrm{mm})$ & $26.97 \pm 0.79$ & $23.09 \pm 0.78$ & $<\mathbf{0 . 0 0 1}$ \\
Kmed $(\mathrm{D})$ & $43.79 \pm 2.04$ & $43.73 \pm 1.61$ & 0.85 \\
GCC superior $(\mu \mathrm{m})$ & $94.90 \pm 13.94$ & $109.30 \pm 9.94$ & $<\mathbf{0 . 0 0 1}$ \\
GCC inferior $(\mu \mathrm{m})$ & $91.55 \pm 14.53$ & $108.32 \pm 9.88$ & $<\mathbf{0 . 0 0 1}$ \\
SSI score & $7.57 \pm 0.08$ & $8.29 \pm 0.10$ & $<\mathbf{0 . 0 0 1}$ \\
\hline BCVA best-con & & &
\end{tabular}

$B C V A$ best-corrected visual acuity, $I O P$ intraocular pressure, $A L$ axial length, $C M T$ central macular thickness, $G C C$ ganglion cell complex, SSI signal strength index.

Bold values indicate statistical significance $p<0.05$.

compared with the controls $(p<0.001)$. Also, SSI score significantly differed between the groups $(p<0.001)$.

RNFL (mean, superior, inferior, and nasal) and ETDRS thicknesses $(p<0.001)$ differed significantly between the two groups (Table 3). The OCT-A findings of the participants are summarized in Table 4 and Fig. 2. Although superficial or deep FAZ did not significantly differ between the two groups, the superficial $(p=0.005)$ and deep 
Table 3 RNFL and macular thickness measurements of study participants.

\begin{tabular}{|c|c|c|c|c|}
\hline & & $\begin{array}{l}\text { High myopia } \\
(n: 92)\end{array}$ & $\begin{array}{l}\text { Control } \\
(n: 70)\end{array}$ & $p$ \\
\hline \multirow[t]{5}{*}{ RNFL $(\mu \mathrm{m})$} & Average & $94.17 \pm 12.12$ & $107.67 \pm 12.67$ & $<0.001$ \\
\hline & Superior & $111.07 \pm 27.13$ & $135.78 \pm 19.85$ & $<0.001$ \\
\hline & Nasal & $66.68 \pm 22.57$ & $78.21 \pm 16.84$ & $<0.001$ \\
\hline & Inferior & $116.00 \pm 22.37$ & $139.02 \pm 20.36$ & 0.001 \\
\hline & Temporal & $77.31 \pm 20.32$ & $77.90 \pm 15.81$ & 0.97 \\
\hline \multirow{4}{*}{$\begin{array}{l}\text { ETDRS outer } \\
\text { ring } \\
\text { thicknesses } \\
(6 \mathrm{~mm})(\mu \mathrm{m})\end{array}$} & Superior & $291.09 \pm 31.24$ & $331.45 \pm 18.45$ & 0.001 \\
\hline & Nasal & $300.34 \pm 39.21$ & $342.60 \pm 19.03$ & 0.001 \\
\hline & Inferior & $287.42 \pm 30.13$ & $331.85 \pm 20.22$ & 0.001 \\
\hline & Temporal & $278.21 \pm 44.59$ & $323.14 \pm 19.32$ & 0.001 \\
\hline \multirow{4}{*}{$\begin{array}{l}\text { ETDRS inner } \\
\text { ring } \\
\text { thicknesses } \\
(3 \mathrm{~mm})(\mu \mathrm{m})\end{array}$} & Superior & $323.15 \pm 30.67$ & $347.11 \pm 18.32$ & 0.001 \\
\hline & Nasal & $317.82 \pm 39.60$ & $344.20 \pm 16.96$ & 0.001 \\
\hline & Inferior & $306.97 \pm 39.20$ & $342.28 \pm 18.75$ & 0.001 \\
\hline & Temporal & $301.22 \pm 46.80$ & $329.61 \pm 16.34$ & 0.001 \\
\hline $\mathrm{CMT}(\mu \mathrm{m})$ & & $268.59 \pm 39.41$ & $261.25 \pm 20.52$ & 0.29 \\
\hline
\end{tabular}

$R N F L$ retinal nerve fiber layer thickness, ETDRS score of Early Treatment Diabetic Retinopathy Study (macular thickness map).

Bold values indicate statistical significance $p<0.05$.

Table 4 OCT-angiography findings of study participants.

\begin{tabular}{llll}
\hline & $\begin{array}{l}\text { High myopia } \\
(n: 92)\end{array}$ & $\begin{array}{l}\text { Control } \\
(n: 70)\end{array}$ & $p$ \\
\hline FAZ superficial $\left(\mathrm{mm}^{2}\right)$ & $0.31 \pm 0.06$ & $0.33 \pm 0.11$ & 0.23 \\
Superficial VD $(\%)$ & $34.8 \pm 3.5$ & $36.5 \pm 4.2$ & $\mathbf{0 . 0 0 5}$ \\
FAZ deep $\left(\mathrm{mm}^{2}\right)$ & $0.47 \pm 0.08$ & $0.49 \pm 0.14$ & 0.28 \\
Deep VD $(\%)$ & $39.4 \pm 5.3$ & $42.6 \pm 4.5$ & $\mathbf{0 . 0 0 1}$ \\
\hline
\end{tabular}

$F A Z$ foveal avascular zone, $V D$ retinal microvessel density.

Bold values indicate statistical significance $p<0.05$.

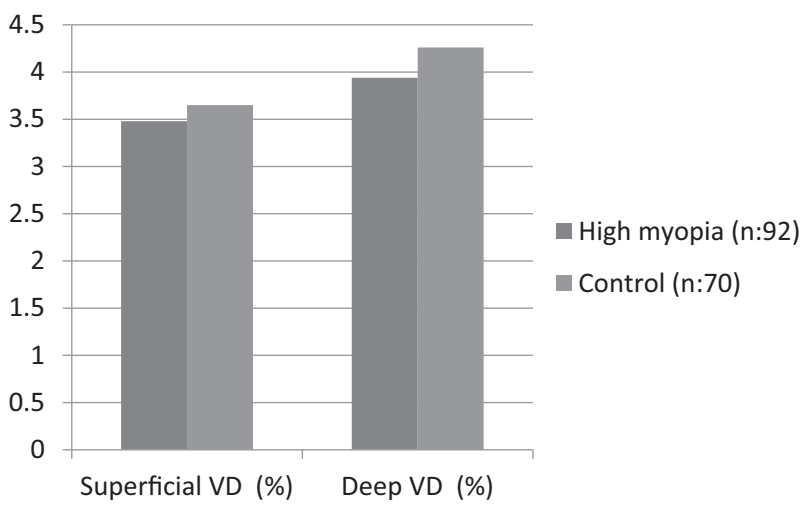

Fig. 2 Comparison of vascular density between groups.

$(p<0.001)$ vascular densities values were significantly lower in the high myopia group compared with the control cases (Fig. 3).
In the correlation analysis, there was a negative correlation between age and AL and both SVD and DVD, while there were significant positive correlations between RNFL, GCC and BCVA and SVD and DVD. In the multivariate regression analysis of the SVD, we first excluded superficial and deep FAZ, Kmed, and CMT due to lack of statistical significance. We then excluded the superior, nasal, inferior and temporal RNFL, ETDRS and GCC thicknesses due to the collinearity with the average RNFL thickness, followed by the exclusion of the SE due to the collinearity with the AL. In the final model (regression coefficient, $r^{2}=0.200$ ), a greater SVD was independently associated with younger age and shorter AL and thicker average RNFL thickness $(P<0.001,=0.035$, and 0.049 respectively $)$. Same regression model was repeated for the DVD (regression coefficient, $r^{2}=0.288$ ). A greater DVD was independently associated with the younger age, better VA (smaller logMAR value $)$ and shorter $\operatorname{AL}(P<0.001,=0.046$, and 0.043 respectively, Table 5).

\section{Discussion}

In this study, we determined a significant decrease in the RNFL thickness on the superior, nasal, and inferior regions in patients with high myopia compared with the control cases. The ETDRS scores were also significantly decreased in high myopia cases for all quadrants. Regarding the angiographical findings, although there was not significant alteration in the FAZ values, the microvessel density was lower in both superficial and deep layers for the patients with high myopia. Based on the correlation analysis, this alterations in SVD and DVD were more prominent with an elongation in $\mathrm{AL}$ and a decrease in RNFL and GCC thicknesses. Moreover, there were significant positive correlations between the BCVA and SVD and DVD. Our findings are compatible with the recent results reported in the literature. It has been suggested that with the progress of myopia, the eyeball elongates, mechanically stretching the retinal tissue and causing a decrease in the RNFL thickness and retinal microvascular density $[15,16]$. Yang et al. [17] reported that SVD and DVD were significantly lessened in patients with high myopia. They also reported a negative correlation between AL and SVD and DVD. Fan et al. [18] analysed the OCT-angiography results of a sample consisting of a control group and moderate and high myopia cases. They reported that both SVD and DVD were decreased in the myopia groups. In this study, SVD and DVD were reported to be associated with AL and SVD was associated with the GCC thickness. Milani et al. [19] also determined that although FAZ were similar between the myopic and control eyes, the SVD was significantly 
Fig. 3 OCT-A image of the superficial and deep capillary plexus of the control group (at the top) and myopic patient (at the bottom). Reduced vessel density is observed in the myopia group.
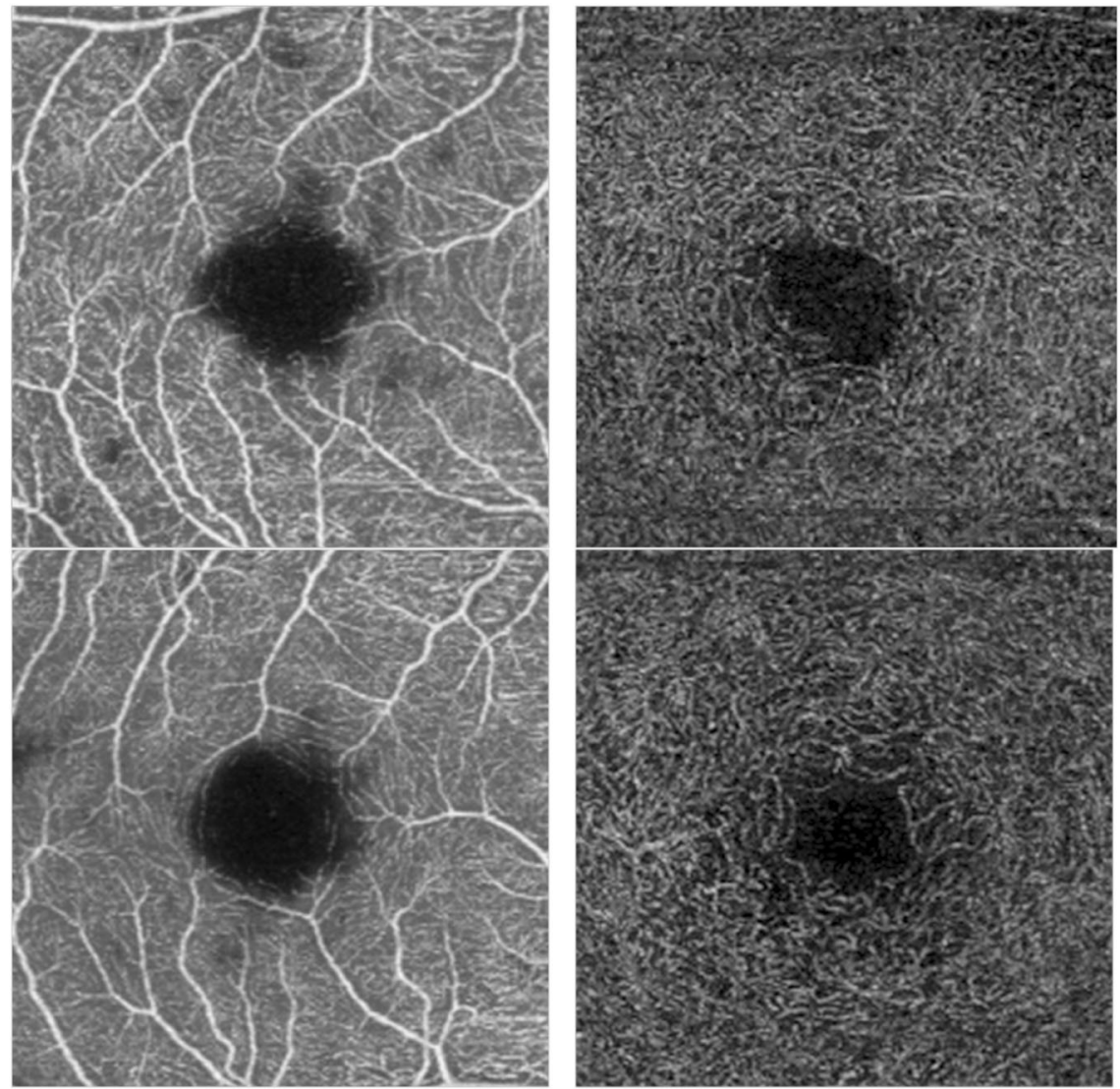

Table 5 Final multivariate regression models for SVD and DVD.

\begin{tabular}{llccc}
\hline & \multicolumn{1}{l}{$B$} & \multicolumn{1}{l}{$95 \%$ CI } & $P$ \\
\hline SVD & Age & -0.91 & $-0.136 /-0.046$ & $<\mathbf{0 . 0 0 1}$ \\
& SSI score & -0.088 & $0.718 / 0.541$ & 0.782 \\
& BCVA (LogMAR) & -8.370 & $-19.935 / 3.196$ & 0.618 \\
& RNFL average & 0.043 & $-0.003 / 0.089$ & $\mathbf{0 . 0 4 9}$ \\
& AL & -0.161 & $-0.516 / 0.137$ & $\mathbf{0 . 0 3 5}$ \\
DVD & Age & -0.128 & $-0.184 /-0.071$ & $<\mathbf{0 . 0 0 1}$ \\
& SSI score & -0.235 & $-1.028 / 0.559$ & 0.56 \\
& BCVA (LogMAR) & -14.358 & $-29.432 /-0.275$ & $\mathbf{0 . 0 4 6}$ \\
RNFL average & 0.054 & $-0.003 / 0.112$ & 0.065 \\
AL & -0.044 & $-0.882 / 0.004$ & $\mathbf{0 . 0 4 3}$ \\
\hline
\end{tabular}

$S V D$ superficial retinal vascular densities, $D V D$ deep retinal vascular densities, $S S I$ signal strength index, $B C V A$ best-corrected visual acuity, $R N F L$ retinal nerve fiber layer thickness, $A L$ axial length.

Bold values indicate statistical significance $p<0.05$.

lower. They also reported a significant correlation between the SVD and retinal thickness. Recently, Qu et al. [20] reported that in high myopia cases, there were significant thinning in the average RNFL and focal thinning of RNFL in the temporal, superior, and inferior quadrants compared with the healthy controls. The authors also noted that although there were no significant differences in the retinal blood flow velocities of patients with moderate or high myopia compared with the healthy controls, both SVD and DVD were significantly lower in the myopia groups compared with the control group. Similar results were obtained by Li et al. [15] who also reported a negative correlation between AL and SVD and DVD. Our results were consistent with the literature in that; we determined a significant decrease in SVD and DVD, which was significantly associated with an elongation in AL and a decrease in RNFL and GCC thicknesses.

We determined a negative correlation between age and SVD and DVD. Similarly, Leng et al. [21] reported that the vessel densities of the superficial and deep capillary plexuses were negatively correlated with age. Supporting our results, Leveziel et al. [22], who used fluorescein angiography and spectral-domain OCT in highly myopic patients also found that the choroidal neovascularization area was significantly smaller in younger patients ( $<55$ years) than in older patients. In the current study, we also determined a negative correlation between logMAR converted BCVA and vascular densities, which indicates the importance of alterations in vascular structures for the visual acuity of patients. In a similar way, Mo et al. [23] reported a positive correlation 
between BCVA and the superficial and deep macular flow densities. Thus, we consider that since high myopia is an important etiological factor for visual impairment, ophthalmologists should be made aware of the association between vascular changes and visual disturbances.

In the present study we compared the SSI score between the groups. It is common to experience that the quality of OCT and OCT-A scans in high myopic patients can severely inferior compared with those of healthy individuals. Even though we only included the scans with SSI score $>6$, in the high myopia group the SSI score was significantly lesser than the healthy controls. However, after the multivariate regression analysis the greater VD of both superficial and deep retinal plexus uses were remained associated with the shorter $\mathrm{AL}$ independent from the SSI score.

OCT-A is a noninvasive method for defining vascular alterations at the retinal level. In a validity and reliability analysis, Miyata et al. reported that OCT-A was able to detect most myopic choroidal neovascularizations with high-quality images and eliminated the necessity of conventional fundus fluorescein angiography [24].

The present study is the first in our knowledge that investigated microvascular network in high myopic eyes with the Nidek's RS3000 Advance. Although the results of our study are consistent with the results of other studies, vascular density values are observed to be very different from other studies [9]. Therefore, it is very important that each device should have its own database for different pathologies.

There are some limitations to this study that should be mentioned. For example, the comparison of the results with patients having a moderate degree of myopia was not undertaken, but this could be helpful in identifying vascular alterations at different stages of myopia. Although the results of correlation analyses helped us identify the presence of an association of vascular alterations with increased AL and decreased RNFL and GCC thicknesses, the mechanism of decreased macular vascular density in high myopia remains unclear. Another limitation is since this is an exploratory study, no adjustment of multiple comparisons were made, therefore it is impossible for us to draw definite conclusions.

In conclusion, in patients with high myopia, the superficial and deep retinal vascular densities in the macular region decreased with an increase in $\mathrm{AL}$ and a decrease in RNFL and GCC thicknesses. Moreover, there was a significant correlation between BCVA and retinal vascular densities. These vascular alterations may be one of the main pathophysiological mechanisms in high myopia, and alternative treatments and preventive measures targeting these vascular alterations may be the subject of future studies.

\section{Summary}

\section{What was known before}

- High myopia can affect the macula, leading to a dramatic reduction in visual acuity. Therefore, it is highly important for ophthalmologists to determine macular changes associated with high myopia. Since high myopia can dramatically damage visual acuity, the findings of OCT-A are important to elucidate the pathophysiological mechanisms.

\section{What this study adds}

- In patients with high myopia, the superficial and deep retinal vascular densities in the macular region decreased with an increase in $\mathrm{AL}$ and a decrease in RNFL and GCC thicknesses. Vascular alterations may be one of the main pathophysiological mechanisms in high myopia, and alternative treatments and preventive measures targeting these vascular alterations may be the subject of future studies.

\section{Compliance with ethical standards}

Conflict of interest The authors declare that they have no conflict of interest.

Publisher's note Springer Nature remains neutral with regard to jurisdictional claims in published maps and institutional affiliations.

\section{References}

1. Holden BA, Fricke TR, Wilson DA, Jong M, Naidoo KS, Sankaridurg P, et al. Global Prevalence of Myopia and High Myopia and Temporal Trends from 2000 through 2050. Ophthalmology. 2016;123:1036-42.

2. Siegwart JT Jr, Norton TT. Perspective: how might emmetropization and genetic factors produce myopia in normal eyes? Optom Vis Sci. 2011;88:E365-72.

3. Kumar A, Chawla R, Kumawat D, Pillay G. Insight into high myopia and the macula. Indian J Ophthalmol. 2017;65:85-91.

4. Morgan IG, Ohno-Matsui K, Saw SM. Myopia. Lancet. 2012;379:1739-48.

5. Silva R. Myopic maculopathy: a review. Ophthalmologica. 2012;228:197-213.

6. Kamal Salah R, Morillo-Sánchez MJ, García-Ben A, Rius-Diaz F, Cilveti-Puche Á, Figueroa-Ortiz L, et al. The effect of peripapillary detachment on retinal nerve fiber layer measurement by spectral domain optical coherence tomography in high myopia. Ophthalmologica. 2015;233:209-15.

7. Li H, Mitchell P, Rochtchina E, Burlutsky G, Wong TY, Wang JJ. Retinal vessel caliber and myopic retinopathy: the blue mountains eye study. Ophthalmic Epidemiol. 2011;18:275-80. 
8. De Carlo T, Romano A, Waheed N, Duker J. A review of optical coherence tomography angiography (OCTA). Int J Retin Vitreous. 2015;1:1-15.

9. Corvi F, Pellegrini M, Erba S, Cozzi M, Staurenghi G, Giani A. Reproducibility of vessel density, fractal dimension, and foveal avascular zone using 7 different optical coherence tomography angiography devices. Am J Ophthalmol. 2018; 186:25-31.

10. Al-Sheikh M, Phasukkijwatana N, Dolz-Marco R, Rahimi M, Iafe $\mathrm{N}$, Freund B, et al. Quantitative OCT angiography of the retinal microvasculature and the choriocapillaris in myopic eyes. Investig Ophthalmol Vis Sci. 2017;58:2063-9.

11. Ohno-Matsui K, Kawasaki R, Jonas JB, Cheung CW, Saw SM, Verhoeven VJ, et al. for the metanalysis for pathological myopia (META-PM) study group. International photographic classification and grading system for myopic maculopathy. Am J Ophthalmol. 2015;159:877-83.

12. Abdolrahimzadeh S, Parisi F, Plateroti AM, Evangelista F, Fenicia V, Scuderi G, et al. Visual acuity, and macular and peripapillary thickness in high myopia. Curr Eye Res. 2017; 42:1468-73.

13. Early Treatment Diabetic Retinopathy Study design and baseline patient characteristics. ETDRS report number 7. Ophthalmology. 1991;98 Suppl 5:741-56.

14. Campbell JP, Zhang M, Hwang TS, Bailey ST, Wilson DJ, Jia Y, et al. Detailed vascular anatomy of the human retina by projection-resolved optical coherence tomography angiography. Sci Rep. 2017;7:42201.

15. Li M, Yang Y, Jiang H, Gregori G, Roisman L, Zheng F, et al. Retinal microvascular network and microcirculation assessments in high myopia. Am J Ophthalmol. 2017;174:56-67.
16. Azemin MZ, Daud NM, Ab Hamid F, Zahari I, Sapuan AH. Influence of refractive condition on retinal vasculature complexity in younger subjects. Sci World J. 2014;2014:783525.

17. Yang Y, Wang J, Jiang H, Yang X, Feng L, Hu L, et al. Retinal microvasculature alteration in high myopia. Investig Ophthalmol Vis Sci. 2016;57:6020-30.

18. Fan H, Chen HY, Ma HJ, Chang Z, Yin HQ, Ng DS, et al. Reduced macular vascular density in myopic eyes. Chin Med J. 2017;130:445-51.

19. Milani P, Montesano G, Rossetti L, Bergamini F, Pece A. Vessel density, retinal thickness, and choriocapillaris vascular flow in myopic eyes on OCT angiography. Graefes Arch Clin Exp Ophthalmol. 2018;256:1419-27.

20. Qu D, Lin Y, Jiang H, Shao Y, Shi Y, Airen S, et al. Retinal nerve fiber layer (RNFL) integrity and its relations to retinal microvasculature and microcirculation in myopic eyes. Eye Vis. 2018;5:25.

21. Leng Y, Tam EK, Falavarjani KG, Tsui I. Effect of age and myopia on retinal microvasculature. Ophthalmic Surg Lasers Imaging Retin. 2018;49:925-31.

22. Leveziel N, Caillaux V, Bastuji-Garin S, Zmuda M, Souied EH. Angiographic and optical coherence tomography characteristics of recent myopic choroidal neovascularization. Am J Ophthalmol. 2013;155:913-9.

23. Mo J, Duan A, Chan S, Wang X, Wei W. Vascular flow density in pathological myopia: an optical coherence tomography angiography study. BMJ Open. 2017;7:e13571.

24. Miyata M, Ooto S, Hata M, Yamashiro K, Tamura H, AkagiKurashige Y, et al. Detection of myopic choroidal neovascularization using optical coherence tomography angiography. Am J Ophthalmol. 2016;165:108-14. 